\title{
Croissance du trèfle violet (Trifolium pratense L.) en association avec du ray-grass d'Italie (Lolium multiflorum Lam. ssp. italicum) : II. - Démographie des organes, biomasse et interférences biologiques

\author{
Jean-Paul MAITRE, Louis ASSEMAT $\left({ }^{*}\right)$ \& Pierre JACQUARD $\left(^{*}\right)(2)$ \\ Ecole Nationale d'Ingénieurs des Travaux agricoles, Chaire d'Agronomie, 21, bd O. de Serres, B.P. 48, \\ F 21800 Quetigny \\ (*) C.N.R.S., Unité de Biologie des Populations et des Peuplements, Centre Louis Emberger, B.P. 5051, \\ Route de Mende, F 34033 Montpellier Cedex.
} temps, des relevés météorologiques ont été réalisés. Après analyse et interprétation des résultats, il se confirme que la densité modifie l'organisation démographique à l'échelle de l'individu. L'absence d'interactions densité $x$ proportion est à noter. Parmi les aspects démographiques, on relève que, chez le trèfle, les effectifs diminuent en $2^{\mathrm{e}}$ année. A 100 plantes par $\mathrm{m}^{2}$ et quelle que soit la coupe, les feuilles naissent beaucoup plus tôt et meurent aussi plus tôt qu'à densité élevée. A faible densité, la ramification est plus précoce. Une proportion croissante de ray-grass d'Italie agit comme la densité. Pour relier variables climatiques et paramètres démographiques, on a utilisé les relevés journaliers. Les paramètres de mortalité par exemple ont été étudiés sur la base d'un individu " type ». La température a un effet sur le taux d'émission des feuilles. Pour ce qui a trait à la biomasse, à faible densité, celle du trèfle est constituée surtout de tiges et de limbes épais. A moyenne et forte densité, les limbes sont minces. On note une biomasse supérieure des associations par rapport aux cultures pures. La proportion de 50 p. 100 de trèfle fournit le meilleur résultat. Dans ces conditions, l'équilibre à la récolte peut être considéré comme bon d'un point de vue zootechnique. En ce qui concerne les interférences biologiques, le ray-grass est avantagé, proportionnellement à la contribution de la légumineuse. D'après la somme des rendements relatifs, les interférences sont principalement du type compétition et résultent d'une combinaison d'effets intra et interspécifiques.

Mots clés additionnels : Cultures associées, concurrence, feuilles.

\section{SUMMARY}

Associated growth of red clover (Trifolium pratense L.) with Italian rye-grass (Lolium multiflorum Lam. ssp. italicum) : II Demography of organs, biomass and biological interference.

Individual plants of red clover were submitted to measurements concerned mainly with biomass and meteorological data were recorded. After analysis and interpretation of results, it was confirmed that density modifies demographic organization at the individual level. The lack of density $x$ proportion interactions should be stressed. Among demographic aspects, it was significant that, in clover, the number of individuals declined in the second year. At 100 plants per $\mathrm{m}^{2}$ and independently of cutting, the leaves emerged earlier than at high density and so died earlier. At low density, ramification was also earlier. An increasing admixture of grass had the same effect as density. To relate climate and demographic parameters, daily data were used. Mortality was studied on the basis of a standard individual. Temperature had a strong effect on the rate of leaf emergence. At low density, biomass of clover was mainly allocated to stems and thick leaf laminas. At medium and high density, the laminas were thin. There was a higher biomass of mixtures compared to pure stand. The best results were with a proportion of $50 \%$ clover. These conditions gave a good balance at harvest from the nutritive point of view. With respect to biological interference, rye-grass was at an advantage, in direct ratio to the contribution of the legume. According to the RYT, interference was mainly of the competition type, combining intra and interspecific effects.

Additional key words : Mixed cropping, competition, leaf.
(1) Recherche financée en partie par la D.G.R.S.T., décisions d'aide $n^{\circ}$ V.I. 13.77.1861 et 1862 , dans le cadre de l'action incitative "Liaisons Recherche-Enseignement Supérieur dans les disciplines agronomique et vétérinaire ».
(2) Avec la collaboration de J. L. Gaujard et Y. Ballly, étudiants de l'E.N.I.T.A. 


\section{INTRODUCTION}

A la suite de recherches conduites sur du trèfle violet, dans des dispositifs comprenant des associations et les cultures pures, on a pu décrire l'organisation morphologique des plantes (MAITRE et al., 1985), comparer l'état végétatif et l'état reproducteur, apprécier enfin l'effet d'une coupe sur l'organisation ultérieure.

On se propose ici de suivre la démographie des organes, d'estimer la biomasse et ses composantes, d'en déduire la nature des interférences biologiques, à partir de mesures concernant la surface foliaire, le nombre de feuilles, etc... Les relevés climatiques réalisés l'ont été selon les normes de la météorologie nationale.

Rappelons que le choix du matériel végétal a porté sur des cultivars reconnus comme productifs : trèfle violet « Rotra » (tétraploïde), ray-grass d'Italie « Adret » (diploïde, non alternatif).

\section{DÉMOGRAPHIE DES ORGANES}

\section{A. Principe}

Chaque individu de trèfle violet peut être considéré au cours de sa croissance, comme un générateur de feuilles. Celles-ci naissent, émergent puis meurent, ce qui détermine la variation temporelle de la démographie ou cinétique de la population ;

$$
\mathrm{NV}_{\mathrm{t}}=\mathrm{NV}_{\mathrm{t}-1}+\mathrm{B}_{\mathrm{t}}-\mathrm{D}_{\mathrm{t}} \text {. }
$$

avec $\mathrm{NV}_{\mathrm{t}}=$ nombre de feuilles vivantes au temps $\mathrm{t}$.

$B_{t}\left(D_{t}\right)=$ nombre de feuilles émises (ou mortes) au
temps $t$.

Les taux relatifs instantanés d'émission et de mortalité peuvent être estimés par :

$$
\mathrm{b}(\mathrm{t})=\frac{\mathrm{Bt}}{\mathrm{NV}} \quad \text { et } \quad \mathrm{d}(\mathrm{t})=\frac{\mathrm{Dt}}{\mathrm{NV}}
$$

et déterminent le taux instantané relatif de croissance $r_{(t)}$ du nombre de feuilles vivantes :

$$
\frac{1}{N V_{t}} \frac{\Delta N V_{t}}{\Delta t}=r(t)=b(t)-d(t)
$$

\section{B. Mode de calcul}

Ces variables ont été calculées journellement par intrapolation linéaire à partir des observations réalisées sur le terrain dans le cadre de la $2^{\mathrm{e}}$ expérience présentée dans la partie I (MAITRE et al., 1985). La fréquence des observations était comprise entre 1 par jour et 1 tous les $11 \mathrm{j}$ avec une fréquence moyenne d' 1 tous les 3-4 $j$, en fonction de la vitesse de croissance.

La démographie des feuilles secondaires de trèfle violet (et tertiaires pour les coupes $C_{1}$ et $C_{2}$ ) a été étudiée sur 15 plantes (ou modules) par traitement expérimental (densité $\mathrm{x}$ proportion de trèfle violet dans le mélange) pour 4 périodes d'observation :

- Coupe 1, pendant $51 \mathrm{j}$, en juin-juillet 1978 .

- Coupe 2, pendant $36 \mathrm{j}$, en août 1978 .

- Coupe 3, pendant $67 \mathrm{j}$, en avril-mai-juin 1979.

- Coupe 4, pendant $29 \mathrm{j}$, en juin-juillet 1979.

\section{Résultats}

Il semble que la densité détermine l'organisation démographique et la biomasse (matière sèche). Chez le trèfle violet, le nombre d'individus diminue en début de $2^{\mathrm{e}}$ année. A 100 plantes par $\mathrm{m}^{2}$, l'individu se ramifie.

\section{Influence de la densité et de la proportion de trèfle violet}

Pour chaque période d'observations (entre 2 coupes), on a testé l'hypothèse que les taux d'émission et de mortalité des feuilles étaient constants. Les taux moyens journaliers ont été soumis à une analyse de variance pour déterminer l'action des facteurs densité et proportion (tabl. 1). L'effet densité est parfois le seul effet discernable. A faible densité, les feuilles naissent en moyenne beaucoup plus tôt. A forte densité, l'individu se ramifie plus tard qu'à faible densité. Les taux d'émission et de mortalité semblent diminuer quand la densité initiale augmente. La graminée agit comme la densité sur l'organisation de la légumineuse. Il n'y a pas d'interactions entre les 2 facteurs. En $1^{\text {re }}$ année, les taux d'émission sont plus élevés et les taux de mortalité moins élevés qu'en $2^{\mathrm{e}}$ année, ce qui pourrait être une manifestation biologique de la sénescence.

\section{Concurrence intra-plante et facteurs climatiques}

Ceci ne constitue qu'une intégration très limitée des facteurs climatiques et qui demanderait à être développée pour l'introduire dans une tentative de modélisation.

L'hypothèse de constance des taux d'émission et de mortalité pendant chaque période doit donc être rejetée. On peut penser que la morphogénèse d'un pied de trèfle violet est sous la dépendance d'un mécanisme

\section{TABLEAU}

\begin{tabular}{|c|c|c|c|c|c|c|c|}
\hline \multirow{2}{*}{\multicolumn{5}{|c|}{$\begin{array}{c}\text { Taux d'émission } \\
\text { (nombre de feuilles émises/jour/plante } \\
\text { ou moduie) } \\
\text { Emergence rate }\end{array}$}} & \multirow{2}{*}{\multicolumn{3}{|c|}{$\begin{array}{l}\text { Analyse } \\
\text { de variance } \\
\text { Analysis } \\
\text { of variance }\end{array}$}} \\
\hline & & & & & & & \\
\hline \multirow{2}{*}{ Coupe } & \multicolumn{3}{|c|}{ Densité plantes $/ \mathrm{m}^{2}$} & \multirow{2}{*}{ Erreur } & \multirow{2}{*}{$\mathrm{P}$} & \multirow{2}{*}{$\mathrm{D}$} & \multirow{2}{*}{$\mathrm{P} \times \mathrm{D}$} \\
\hline & 100 & 400 & 700 & & & & \\
\hline 1 & 0,437 & 0,179 & 0,130 & 0,013 & NS & $* * *$ & NS \\
\hline 2 & 0,746 & 0,145 & 0,117 & 0,030 & NS & $* * *$ & NS \\
\hline 4 & 0,066 & 0,066 & 0,066 & 0,002 & NS & NS & NS \\
\hline 5 & 0,110 & 0,110 & 0,110 & 0,005 & NS & NS & NS \\
\hline \multicolumn{8}{|c|}{ Taux de mortalité (mortality rate) } \\
\hline 1 & 0,016 & 0,016 & 0,016 & 0,018 & NS & NS & NS \\
\hline 2 & 0,031 & 0,013 & 0,008 & 0,006 & NS & $*$ & NS \\
\hline 4 & 0,062 & 0,062 & 0,062 & 0,002 & $*$ & NS & NS \\
\hline 5 & 0,025 & 0,025 & 0,025 & 0,003 & NS & NS & NS \\
\hline
\end{tabular}

Taux moyens journaliers d'émission et de mortalité des feuilles. Mean daily rate of emergence and mortality of leaves.

$\mathbf{P}=$ proportion $; \mathbf{D}=$ densité $; \mathbf{P} \times \mathbf{D}=$ interaction. 
d'auto-régulation (analogue à une concurrence intraplante) modulé par les ressources disponibles dans le milieu, sous l'effet des facteurs climatiques et de la densité. On a donc essayé de prévoir - toutes périodes d'observations confondues - les taux relatifs journaliers d'émission et de mortalité (ces paramètres étant étudiés pour un individu "type ») à l'aide de données concernant :

- la concurrence intra-plante (nombre de feuilles de la plante ou du module),

- la concurrence entre plantes (densité de départ et densité réelle, proportion de trèfle violet dans le mélange),

- le climat (rayonnement global, température moyenne, déficit hydrique ETP-P) ; pour relier ces variables à la démographie, on a utilisé le fichier des relevés journaliers.

Paramètres et variables sont estimés pour chacun des $163 \mathrm{j}$ d'observation dans les 12 traitements expérimentaux ( 3 densités $\times 4$ proportions). Une analyse de régression multiple, pas à pas, sur les 1956 données ainsi obtenues a fourni les résultats de tableau 2 . Etant donné les corrélations étroites existant entre les variables climatiques, l'interprétation biologique de ces régressions est à manier avec une extrême prudence en vue d'une modélisation.

\section{TABLEAU 2}

Analyse progressive de régression linéaire multiple. Progressive analysis of multiple linear regression.

Taux relatif d'émission (Relative rate of emergence)

\begin{tabular}{crr}
\multicolumn{1}{c}{$\mathrm{b}$} & \multicolumn{1}{c}{$\mathrm{s}_{\mathrm{b}}$} \\
\hline$\theta_{3 \mathrm{j}}$ & $221.10^{-5}$ & $19.10^{-5}$ \\
$\mathrm{~N}_{\mathrm{TV} / \mathrm{m}^{2}}$ & $-11.10^{-5}$ & $10^{-5}$ \\
$\mathrm{NV}$ & $-235.10^{-5}$ & $19.10^{-5}$ \\
$\mathrm{~N}_{\mathrm{TV}} / \mathrm{m}^{2} \times \mathrm{P}$ & $8.10^{-5}$ & $10^{-5}$
\end{tabular}

Coefficient de corrélation multiple $=0,474$

Taux relatif de mortalité (Relative mortality rate)

$\begin{array}{crc}\mathrm{N}_{\mathrm{TV} / \mathrm{m}^{2}} & 3 \cdot 10^{-5} & 04 \cdot 10^{-5} \\ \mathrm{P} & -146 \cdot 10^{-4} & 128 \cdot 10^{-5}\end{array}$

Coefficient de corrélation multiple $=0,537$

\begin{tabular}{|c|c|c|}
\hline Variables « explicatives» & (non forcées) & $\begin{array}{c}\text { (« Explanatory " } \\
\text { variables) }\end{array}$ \\
\hline Température du jour $\mathbf{j}$ & $\theta$ & (Daily temperature) \\
\hline Déficit hydrique du jour $\mathrm{j}$ & $\mathrm{dh}$ & $\begin{array}{l}\text { (Water deficit } \\
\text { of the day) }\end{array}$ \\
\hline $\begin{array}{l}\text { Température moyenne des } \\
\text { jours } \mathrm{j}-1, \mathrm{j}, \mathrm{j}+1\end{array}$ & $\theta_{3 \mathrm{j}}$ & (Mean temperature) \\
\hline $\begin{array}{l}\text { Deficit hydrique moyen des } \\
\text { jours } \mathrm{j}-1, \mathrm{j}, \mathrm{j}+1\end{array}$ & & (Mean water deficit) \\
\hline $\begin{array}{l}\text { Nombre de feuilles vivantes/ } \\
\text { plantes }\end{array}$ & $N(V)$ & $\begin{array}{l}\text { (Number of live } \\
\text { leaves/plant) }\end{array}$ \\
\hline $\begin{array}{l}\text { Nombre de pieds (ou de } \\
\text { modules) de trèfle violet } / \mathrm{m}^{2} \text {, } \\
\text { à la coupe }\end{array}$ & & $\begin{array}{l}\text { Number of individuals } \\
\text { (or modules) of red } \\
\text { clover } / \mathrm{m}^{2} \text { at the }\end{array}$ \\
\hline $\begin{array}{l}\text { Proportion de trèfle violet } \\
\text { dans le mélange à la coupe } \\
\left(\mathrm{N}_{\mathrm{TV}} / \mathrm{m}^{2} \times \mathrm{P}\right)\end{array}$ & $\left(N_{T V} / m^{2}\right)$ & $\begin{array}{c}\text { cutting } \\
\text { (Proportion of red } \\
\text { clover in the mixture } \\
\text { at cutting) }\end{array}$ \\
\hline
\end{tabular}

La température est l'élément climatique prépondérant qui influe sur le taux d'émission des feuilles. La mortalité est principalement déterminée par le niveau de la densité.

\section{Durée de vie des feuilles}

Sur les 6686 feuilles observées, 232 seulement ont été utilisables (dates d'émission et de mort connues), c'est-à-dire 3,5 p. 100 de l'échantillon total. Ces feuilles vivent de 8 à 54 j. Sans considérer l'« histoire » de

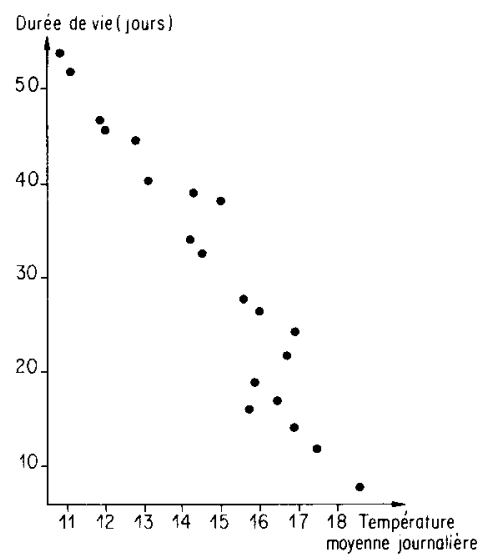

Figure 1

Relation entre la durée de vie (en jours) et la température moyenne journalière.

Relationship between duration of life (in days) and mean daily temperature.

la plante ou du module qui a porté la feuille, on a pu remarquer que la durée de vie d'une feuille est en grande partie explicable par les conditions climatiques moyennes pendant la période de vie de cette feuille (fig. 1) :

Durée de vie $=118,30-5,22 \theta-1,29 \mathrm{dh}$

(j) $\left({ }^{\circ} \mathrm{C} / \mathrm{j}\right)(\mathrm{mm} / \mathrm{j})$

p. 100 d'explication de la somme des carrés: $91,4 \quad 3,1$

Coefficient de corrélation multiple $=0,972$ $(\mathrm{n}=20)$.

Le rayonnement global, très corrélé au déficit hydrique, apporte une information négligeable. En $1^{\text {re }}$ approximation, on pourrait se contenter, dans un modèle, de l'équation de régression linéaire simple de la durée de vie sur la température moyenne journalière.

\section{BIOMASSE}

\section{A. Caractéristiques des feuilles de trèfle violet}

\section{Surface}

Quelle que soit la récolte étudiée, il n'apparaît pas d'effet des traitements, pour une feuille d'ordre déterminé, sauf la $F_{7}$ (1 $1^{\text {re }}$ cohorte) dont la surface est significativement $(\alpha=0,05)$ plus faible à densité élevée :

$F_{7}\left(D_{1}\right)=F_{7}\left(D_{2}\right)=19,8 \mathrm{~cm}^{2}$ contre $F_{7}\left(D_{3}\right)=17,7 \mathrm{~cm}^{2}$, 
TABLEAU 3

Répartition de la matière sèche moyenne produite par un individu (plante ou module) de trèfle violet selon les conditions d'association au champ avec le ray-grass d'Italie.

Allocation of mean dry matter elaborated by an individual (plant of module) of red clover in relation to type of mixture with Italian ryegrass (Field experiment).

\begin{tabular}{|c|c|c|c|c|c|c|c|}
\hline \multirow{2}{*}{\multicolumn{2}{|c|}{ Composante }} & \multicolumn{2}{|c|}{$\mathrm{D}_{1}$} & \multicolumn{2}{|c|}{$\mathrm{D}_{2}$} & \multicolumn{2}{|c|}{$D_{3}$} \\
\hline & & $\begin{array}{l}\text { MS } \\
\text { en } g\end{array}$ & $\%$ & $\begin{array}{l}\text { MS } \\
\text { en } g\end{array}$ & $\%$ & $\begin{array}{l}\text { MS } \\
\text { en } g\end{array}$ & $\%$ \\
\hline \multirow[t]{2}{*}{ Limbes } & $\mathrm{F}_{\mathrm{i}}$ & 0,33 & 11,2 & 0,23 & 38,9 & 0,18 & 47,8 \\
\hline & $\begin{aligned} & f_{i j} \\
+ & f_{i j k}\end{aligned}$ & 0,98 & 32,5 & 0,08 & 13,7 & 0,02 & 5,8 \\
\hline \multicolumn{2}{|l|}{$\mathrm{C}_{1}-$} & 0,73 & 24,5 & 0,26 & 43,3 & 0,17 & 46,2 \\
\hline \multicolumn{2}{|l|}{ Tiges } & 0,95 & 31,8 & 0,02 & 4,1 & 0,001 & 0,2 \\
\hline \multicolumn{2}{|l|}{ Plante } & 2,99 & 100 & 0,59 & 100 & 0,371 & 100 \\
\hline
\end{tabular}

\begin{tabular}{cccccccc}
\hline \hline \multirow{2}{*}{ Limbes } & $\mathrm{f}_{\mathrm{ij}}$ & 0,27 & 33,5 & 0,16 & 38,4 & 0,14 & 38,3 \\
\cline { 2 - 8 } & $\mathrm{f}_{\mathrm{ijk}}$ & 0,03 & 2,5 & 0,01 & 1,6 & 0,01 & 3,1 \\
\hline $\mathrm{C}_{4}$ Pétioles & & 0,15 & 17,3 & 0,11 & 26,6 & 0,11 & 28,3 \\
\hline Tiges & 0,39 & 46,7 & 0,13 & 33,4 & 0,09 & 30,3 \\
\hline Module & 0,83 & 100 & 0,41 & 100 & 0,35 & 100
\end{tabular}

\begin{tabular}{cccccccc}
\hline \multirow{2}{*}{ Limbes } & $\mathrm{f}_{\mathrm{ij}}$ & 0,32 & 28,8 & 0,20 & 33,7 & 0,16 & 38,1 \\
\cline { 2 - 8 } & $\mathrm{f}_{\mathrm{ijk}}$ & 0,05 & 4,5 & 0,02 & 3,0 & 0,01 & 2,4 \\
\hline $\mathrm{C}_{5}$ Pétioles & & 0,14 & 12,6 & 0,12 & 20,0 & 0,10 & 23,8 \\
\hline Tiges & 0,60 & 54,1 & 0,26 & 43,3 & 0,15 & 35,7 \\
\hline Module & 1,11 & 100 & 0,60 & 100 & 0,42 & 100 \\
\hline \hline
\end{tabular}

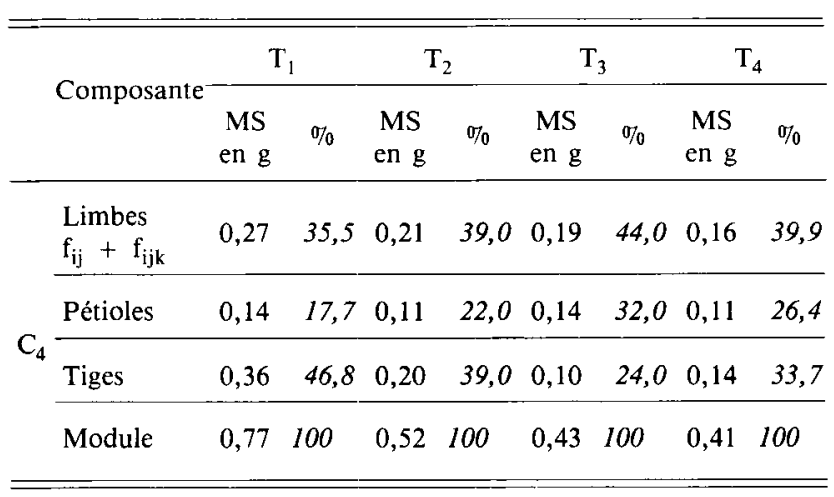

Conditions d'association :

$\mathrm{D}_{1}, \mathrm{D}_{2}, \mathrm{D}_{3}=100,400,700$ plantes $\mathrm{m}^{-2}$

$\mathrm{T}_{1}, \mathrm{~T}_{2}, \mathrm{~T}_{3}, \mathrm{~T}_{4}=25,50,75,100 \%$ de trèfle violet

$\mathrm{C}_{1}, \mathrm{C}_{4}, \mathrm{C}_{5}=1^{\mathrm{re}}, 4^{\mathrm{e}}$ et $5^{\mathrm{e}}$ coupes

$F_{i}, f_{i j}, f_{i j k}=$ feuilles primaires, secondaires, tertiaires. particularité déjà notée, en $1^{\text {re }}$ cohorte, chez des familles consanguines de trèfle lors de travaux antérieurs (MAITRE, non publié). Pour les $1^{\text {res }}$ coupes annuelles $\left(\mathrm{C}_{1}\right.$ et $\left.\mathrm{C}_{4}\right)$, la surface moyenne d'un limbe d'ordre déterminé et homologue est la même. En $\mathrm{C}_{5}$, les limbes présentent une surface régulièrement décroissante avec l'ordre d'émergence. Ceci peut être expliqué par la photopériode : GUY (1964), sur luzerne, relie la croissance en longueur du limbe à cette variable.

\section{Masse surfacique}

L'absence d'interaction que l'on constate pour la plupart des résultats analysés permet une interprétation aisée. Il semble que l'influence de la densité se fasse de plus en plus sentir dans le temps. La modification de l'épaisseur du limbe se fait dans le sens d'un épaississement à faible densité et à faible proportion de trèfle. A moyenne et forte densité, les limbes sont minces.

\section{B. Répartition de la matière sèche à l'échelle de l'indi- vidu de trèfle violet}

La répartition est fortement influencée par la densité aux 3 coupes étudiées (tabl. 3). La densité la plus faible donne toujours les individus les plus lourds, ils sont notamment plus riches en tiges. Les limbes " fils " (secondaires en $\mathrm{C}_{1}$, tertiaires en $\mathrm{C}_{4}$ et $\mathrm{C}_{5}$ ) ont une participation qui augmente lorsque la densité diminue. Comme pour la masse surfacique, il apparaît que les effets des densités $D_{2}$ et $D_{3}$ sont proches. Un effet de la proportion de trèfle qui semble agir sur les composantes du rendement de la même façon que la densité apparaît en 1979. Il convient de mettre en relief à la coupe $C_{1}$, à très forte densité, un phénomène de disparition complète des tiges.

\section{Rendement parcellaire}

D'une façon générale, on note une biomasse supérieure des associations par rapport aux cultures pures de la graminée.

\section{Rendement global : associations et culture pures}

La supériorité de production des associations est imputable à l'insuffisance de la fertilisation azotée par rapport à celle couramment pratiquée $\left(30 \mathrm{~kg} \cdot \mathrm{ha}^{-1}\right.$ d'azote de l'ammonitrate au semis, puis apport de la même dose, après chaque coupe, uniformément sur le dispositif). Cependant les rendements sont équivalents à $18 \mathrm{t} /$ ha avec une répartition quasi égale entre les 2 années. Aucune interaction n'a été mise en évidence (tabl. 4) entre facteurs (densité, proportion), ce qui est à noter et contraire à ce que l'on pourrait supposer. Les différences de rendement n'apparaissent qu'entre les proportions élevées de trèfle et le ray-grass pur. La densité de semis n'influence les rendements qu'en $1^{\text {re }}$ coupe annuelle à cause du ray-grass. 
L'une des explications possibles des valeurs supérieures de biomasse dans les associations graminéelégumineuse pourrait être un apport supplémentaire d'azote au ray-grass. Cependant, si WACQUANT et al. (1981) ont pu mettre en évidence un enrichissement de la rhizosphère en azote minéral durant la nuit, en culture de printemps, lorsque $T$. pratense est associé à une graminée, ce phénomène n'a pu être observé en culture d'automne. Enfin, Chalamet et al. (1983) n'ont pu montrer, malgré l'utilisation de ${ }^{15} \mathrm{~N}^{\prime}$, un éventuel transfert d'azote entre la légumineuse et la graminée.

\section{Rendement de chaque partenaire}

Il est logique, par contre, de mettre en évidence un effet important de la proportion initiale sur le rendement de chaque partenaire. On constate que, pour le trèfle violet, pour 4 récoltes sur 5 , les matières sèches obtenues sont bien distinctes pour chaque proportion.

Ce n'est qu'en $2^{\mathrm{e}}$ année de culture que la production des 2 partenaires est influencée par la densité initiale de semis. Cette dernière s'est modifiée à la suite de l'expansion de chaque espèce, mais aussi de la rigueur de l'hiver qui est à l'origine d'une mortalité élevée. En effet, la démographie des modules parentaux, pendant la $1^{\text {re }}$ année, montre que la mortalité est insignifiante mais, après la $4^{e}$ coupe, les premiers morts sont notés. L'élément déterminant dans les différences observées est la proportion respective des partenaires qui tantôt stimule et tantôt déprime la production de matière sèche du trèfle.

\section{Equilibre et production}

L'équilibre entre les constituants à la récolte est rarement atteint ; cependant, les associations sont moins déséquilibrées que ce que l'on observe habituellement ; la vigueur de " Rotra » est certainement à l'origine de cette tendance. La période automnale $\left(\mathrm{C}_{3}\right)$, particulièrement sèche, a per̨turbé la légumineuse. Seules certaines parcelles ont donné des productions équilibrées ( 40 à $60 \mathrm{p} .100$ de trèfle violet) à 3 coupes sur 5 . Dans les conditions de l'expérience, il apparaît que le meilleur mélange est $50: 50$ à la densité de 400 plantes $/ \mathrm{m}^{2}\left(\mathrm{D}_{2}\right)$. Compte tenu des facultés germinatives des semences, ceci correspond à 9$10 \mathrm{~kg} / \mathrm{ha}^{-1}$ de semences de "Rotra " (1000 graines $=2,82$ à $2,87 \mathrm{~g}$, au seuil $\alpha=0,05$ ) et 8 à $9 \mathrm{~kg} / \mathrm{ha}^{-1} \mathrm{~d}^{\prime} \ll$ Adret » (1 000 graines $=2,17$ à $2,26 \mathrm{~g}$ au seuil $\alpha=0,05$ ).

\section{INTERFÉRENCES BIOLOGIQUES}

Les situations d'interférences peuvent être définies sur la base de leurs manifestations, c'est-à-dire des réponses plastiques (effets biométriques; cf. JACQUARD, 1975). Mais les différentes situations entrấnées par les effets ainsi mesurés :

compétition

opposition

coopération bilatérale

coopération unilatérale correspondent à des processus variables, pour lesquels il faut introduire la notion de concurrence pour l'« espace » de DE WIT (1960). La représentation utilisée par cet auteur permet de décrire les différentes modalités de ce processus (JACQUARD, 1977), grâce notamment à la somme des rendements relatifs (RYT) :

$$
\begin{aligned}
\mathrm{RYT}=\frac{\mathrm{Y}_{\mathrm{i} / \mathrm{j}}}{\mathrm{Y}_{\mathrm{ii}}}+\frac{\mathrm{Y}_{\mathrm{j} / \mathrm{i}}}{\mathrm{Y}_{\mathrm{jj}}} \text { avec } \mathrm{Y}_{\mathrm{i} / \mathrm{j}}=\begin{array}{c}
\text { production de } \mathrm{i} \text { en } \\
\text { présence de } \mathrm{j} .
\end{array} \\
\mathrm{Y}_{\mathrm{ii}}=2 \mathrm{Y}_{\mathrm{i} / \mathrm{i}}
\end{aligned}
$$

Lorsque RYT $>1$, il y a concurrence pour un " espace » partiellement différent ; si RYT $=1$, il y a concurrence pour le même "espace "; enfin, pour RYT $<1$, la croissance a lieu dans des niches partiellement non exploitées.

Ici les valeurs du RYT ont été calculées sur la base des productions de 4 lignes (2 lignes de chaque espèce).

Les figures 2 et 3 rendent compte des interférences rencontrées. Sur la figure 2 , même si parfois la situation observée est à la limite de la compétition ou de la coopération, on a indiqué la tendance globale de la coupe $\left(T_{2}\right.$ et $T_{3}$ en $C_{1}$ et $\left.C_{4}\right)$. Malgré la faible performance des cultures pures de ray-grass d'Italie, les rendements relatifs de cette espèce sont supérieurs à 0,5 : elle est avantagée. Cet avantage est proportionnel à la contribution de la légumineuse : il est relativement plus apparent pour la proportion de 75 p. 100 de trèfle.

Dans les conditions de l'expérience (champ), il est évident que la compétition mise en évidence dans le cas de la coupe $\mathrm{C}_{3}$ n'est que la résultante d'une contrainte hydrique imposée au trèfle violet. Il est remarquable que le trèfle violet ne soit affecté par la présence du rays-grass d'Italie que dans 2 cas sur 5 .

L'absence d'interaction dans l'analyse détaillée de la variance des résultats de matière sèche permet d'assimiler tout effet du traitement densité à une interférence de type intraspécifique et un effet du traitement proportion à une interférence de type interspécifique (tabl. 4). Lorsque les 2 effets existent simultanément, l'une des 2 interférences amplifie l'autre sans qu'il y ait interaction au sens statistique.

D'après la somme des rendements relatifs, les interférences sont du type coopération ou compétition. Ces dernières sont fréquentes au profit de la graminée. Les premières semblent induites essentiellement par les conditions de milieu, même quand elles semblent dépendre de la structure génotypique utilisée (MAITRE et al., 1985).

\section{CONCLUSION GÉNÉRALE}

L'une des voies de construction d'un modèle de fonctionnement est celle qui repose sur une base morphogénétique précise, permettant une bonne connaissance des modalités de la production à partir des matériaux primaires $C$ et $N$, non envisagés ici. Cette voie semble particulièrement adaptée au cas des associations fourragères (JACQUARD, 1975, 1977). Elle nécessite des études préliminaires dont certaines ont 
TABLEAU 4

Signification, classement et équation de réponse de la production de matière sèche parcellaire et de chaque partenaire selon les conditions d'association au champ.

Significance, ranking and response equation for dry matter production/plot and for production/component in relation type of mixture (field experiment).

\begin{tabular}{|c|c|c|c|c|c|c|}
\hline & & \multicolumn{5}{|c|}{ Source de variation } \\
\hline \multicolumn{2}{|r|}{$\begin{array}{l}\text { Production } \\
\text { en M.S. }\end{array}$} & \multicolumn{2}{|r|}{ Densité (d) } & \multicolumn{2}{|r|}{ Proportion (p) } & \multirow[t]{2}{*}{$\begin{array}{l}\text { Interaction } \\
\quad \mathrm{d} \times \mathrm{p}\end{array}$} \\
\hline \multirow{3}{*}{$\mathrm{C}_{1}$} & Globale & $\mathrm{S}$ & $\frac{D_{3} D_{2} D_{1}}{589.10^{-4} d+415}$ & HS & $\begin{array}{l}\frac{\mathrm{T}_{4} \mathrm{~T}_{3} \mathrm{~T}_{2}}{\mathrm{~T}_{1} \mathrm{~T}_{0}} \\
-0,025 \mathrm{p}^{2}+0,45 \mathrm{p}+326,92\end{array}$ & \\
\hline & TV & - & & HS & $\begin{array}{l}\mathrm{T}_{4} \mathrm{~T}_{3} \mathrm{~T}_{2} \mathrm{~T}_{1} \\
5,36 \mathrm{p}-26,48\end{array}$ & - \\
\hline & RGI & - & & HS & $\begin{array}{l}\mathrm{T}_{0} \mathrm{~T}_{1} \mathrm{~T}_{2} \mathrm{~T}_{3} \\
-2,20 \mathrm{p}+344,42\end{array}$ & - \\
\hline \multirow{3}{*}{$\mathrm{C}_{2}$} & Globale & - & & $\mathrm{HS}$ & $\frac{\mathrm{T}_{2} \mathrm{~T}_{3} \mathrm{~T}_{4} \mathrm{~T}_{1}}{-320,33 \mathrm{p}^{2}}+452,10$ p 303,48 & - \\
\hline & TV & - & & HS & $\begin{array}{l}T_{4} T_{3} T_{2} T_{1} \\
398,84 p+34,07\end{array}$ & - \\
\hline & RGI & - & & HS & $\begin{array}{l}\frac{T_{0} T_{1}}{-220} T_{2} T_{3} \\
-48 p+311,67\end{array}$ & - \\
\hline \multirow{3}{*}{$\mathrm{C}_{3}$} & Globale & - & & $\mathrm{HS}$ & $\frac{\mathrm{T}_{0} \mathrm{~T}_{1} \mathrm{~T}_{2} \mathrm{~T}_{3} \mathrm{~T}_{4}}{-159,41 \overline{\mathrm{p}}+384,86}$ & - \\
\hline & $\mathrm{TV}$ & - & & HS & $\frac{\mathrm{T}_{4}}{227,06 \overline{\mathrm{p}}+33,38}$ & - \\
\hline & RGI & - & & $\mathrm{HS}$ & $\begin{array}{l}\mathrm{T}_{0} \mathrm{~T}_{1} \mathrm{~T}_{2} \mathrm{~T}_{3} \\
-282,86 \mathrm{p}+378,98\end{array}$ & - \\
\hline \multirow{3}{*}{$\mathrm{C}_{4}$} & Globale & HS & $\underline{\mathrm{D}_{2} \mathrm{D}_{3}} \mathrm{D}_{1}$ & HS & $\frac{T_{3} T_{4} T_{2} T_{1}}{(1)} T_{0}$ & - \\
\hline & TV & - & & $\mathrm{HS}$ & $\mathrm{T}_{4} \mathrm{~T}_{3} \mathrm{~T}_{2} \mathrm{~T}_{1}$ & - \\
\hline & RGI & $\mathrm{S}$ & $\underline{\mathrm{D}_{3} \mathrm{D}_{2}} \mathrm{D}_{1}$ & HS & $\frac{T_{0} T_{1}}{(1)} T_{2} T_{3}$ & - \\
\hline \multirow{3}{*}{$\mathrm{C}_{5}$} & Globale & - & & HS & $\frac{\mathrm{T}_{3} \mathrm{~T}_{4} \mathrm{~T}_{2}}{(1)} \mathrm{T}_{1} \mathrm{~T}_{0}$ & - \\
\hline & TV & HS & $\underline{\mathrm{D}_{1} \mathrm{D}_{2}} \mathrm{D}_{3}$ & $\mathrm{HS}$ & $\begin{array}{l}\mathrm{T}_{4} \mathrm{~T}_{3} \mathrm{~T}_{2} \mathrm{~T}_{1} \\
(1)\end{array}$ & - \\
\hline & RGI & $\mathrm{HS}$ & $\underline{\mathrm{D}_{3} \mathrm{D}_{2}} \mathrm{D}_{1}$ & HS & $\frac{\mathrm{T}_{0} \mathrm{~T}_{1} \mathrm{~T}_{2}}{(1)} \mathrm{T}_{3}$ & - \\
\hline
\end{tabular}

Conditions d'association :

$D_{1}, D_{2}, D_{3}=100,400,700$ plantes $m^{-2}$

$\mathrm{T}_{0}, \mathrm{~T}_{1}, \mathrm{~T}_{2} \mathrm{~T}_{3}, \mathrm{~T}_{4}=0,25,50,75,100 \%$ de Trèfle violet

$\mathrm{C}_{1}, \mathrm{C}_{2}, \mathrm{C}_{3}, \mathrm{C}_{4}=1^{\mathrm{re}}, 2^{\mathrm{e}}, \ldots$ coupes

$\mathrm{TV}=$ Trèfle violet

RGI = Ray grass d'Italie

- $\quad=$ absence de signification au seul $\alpha=0,05$

$\mathrm{S}=$ significatif au seuil $\alpha=0,05$

HS $=$ significatif au seuil $\alpha=0,01$

Classement décroissant des moyennes par le test de Newman et Keuls :

$$
\begin{aligned}
& \text { au seuil } \alpha=0,05 \\
& \text { au seul } \alpha=0,01
\end{aligned}
$$

(1) Après l'hiver 78-79, l'évolution des proportions et des densités initiales n'a pas permis d'envisager valablement l'ajustement statistique à une courbe de réponse. 


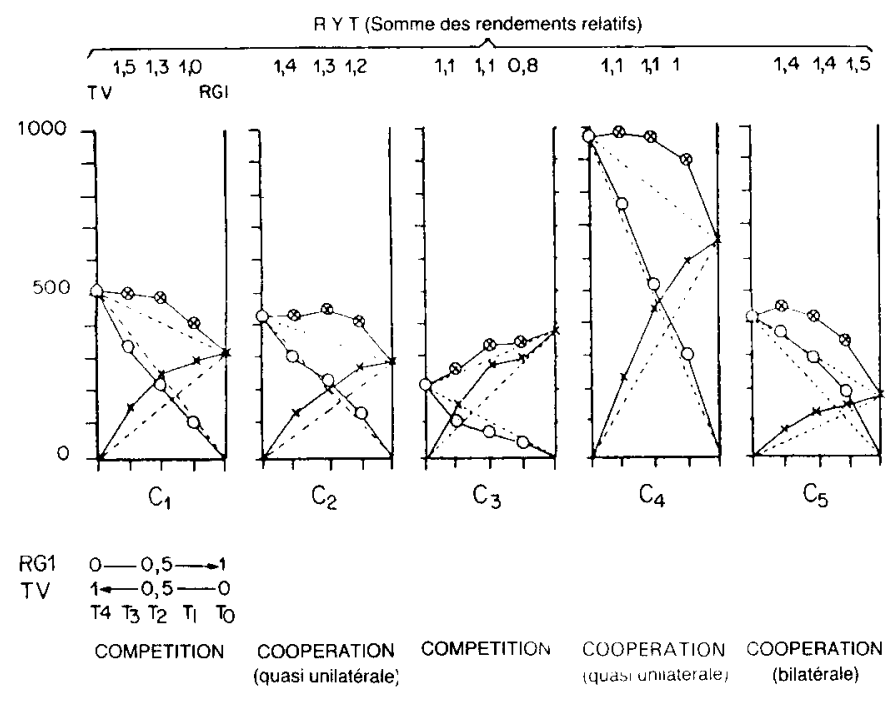

Figure 2

Trèfle violet. Red clover.

$\mathrm{X}$ Ray-grass d'Italie. Italian Rye-grass.

$\otimes$ Association. Association.

- - Production théorique. Theorical production.

Représentation (selon le modèle de $D E$ WIT) des résultats de matière sèche (en $\mathrm{g}$ ) et des situations entraînées par l'effet d'association selon la proportion des partenaires et selon les coupes $\left(C_{1}, C_{2}, C_{3}\right.$, $\left.C_{4}, C_{5}\right)$. Moyenne de 3 blocs, densités confondues.

Presentation (according to DE WIT's model) of results for dry matter (in $\mathrm{g}$ ) and of the situations determined by the effect of association, in relation to the proportion of the partners and to cutting. Mean for 3 blocks, densities confounded. $T_{0}, T_{1}, T_{2}, T_{3}, T_{4}=0,25,50$, $75,100 \mathrm{p} .100$ de trèfle violet. $C_{1}, C_{2}, C_{3}, C_{4}, C_{5}=1^{r e} \ldots 5^{e}$ coupe de l'essai.

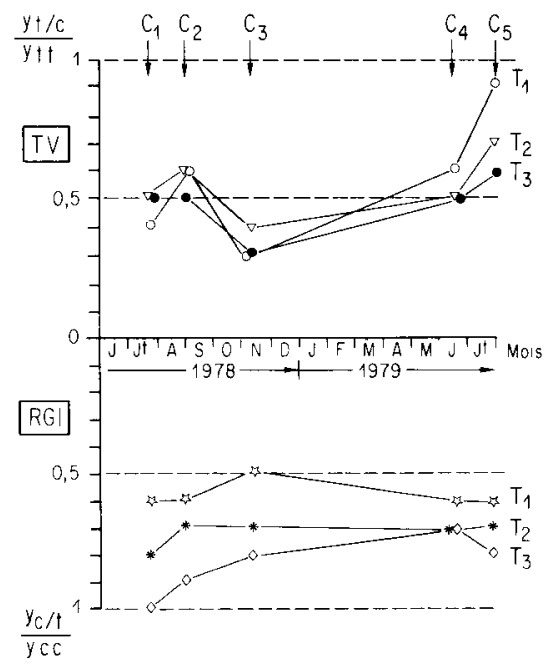

Figure 3

Evolution des rendements relatifs des 2 partenaires pendant 2 années de culture au champ selon les proportions initiales de semis.

Change with time of the relative yields of the two components for two years of field cropping in relation to the starting proportions at sowing.

$\frac{Y_{t / c}}{Y_{t}}=$ Rendement relatif du trèfle violet $\mathrm{cv}$. 《Rotra » $(\mathrm{t})$

$\frac{Y_{t / c}}{Y_{t t}}$ (relative yield of red clover)

$\underline{Y_{c / t}}=$ Rendement relatif du rav-grass d'Italie cv. "Adret" (c) $\overline{Y_{c c}}$ (relative yield of Italian rye-grass)

$\mathrm{C}_{1}, \mathrm{C}_{2}, \mathrm{C}_{3}, \mathrm{C}_{4}, \mathrm{C}_{5}=1^{\mathrm{re}}, 5^{\mathrm{e}}$ coupes de l'essai (first, fifth cutting)

$T_{1}, T_{2}, T_{3}=25,50,75 \mathrm{p} .100$ de trèfle violet. été présentées ici, pour l'association trèfle violet-raygrass d'Italie. Des études antérieures avaient permis de caractériser les effets de groupe dans cette association sans que soit abordée la dynamique de l'élaboration du rendement (MAITRE, 1977). C'est pourquoi on a suivi ici la variation dans le temps de l'organisation foliaire, base du rendement. Cependant, l'interprétation des données recueillies ne permet qu'une extension prudente des conclusions dans les domaines biologique et agronomique.

A la suite de résultats obtenus dans 3 expériences différentes, il a donc été possible de définir l'unité d'organisation du trèfle ou module, ce qui facilite le déroulement d'un travail dynamique et clarifie l'interprétation des résultats. Un individu de trèfle violet peut être considéré comme une tribu de modules de diverses catégories, constituant des ensembles embô̂tés, issus d'un module parental qui, en $1^{\text {re }}$ pousse, est toujours un module primaire. Suivant le cycle de croissance ou de développement imposé par le nombre de récoltes, on peut distinguer :

- une cohorte d'installation,

- des cohortes issues du « démarrage » des modules épargnés par une coupe.

Cette conception modulaire confère au trèfle violet une analogie avec la graminée. Elle suggère également des voies d'approche complémentaires des phénomènes de croissance. Par ailleurs, il semble que l'existence chez la plante d'un stade "ramification » soit à considérer dans l'amélioration de l'aptitude à la concurrence. Enfin une bonne connaissance de la mise en place de l'organisation devrait permettre d'introduire la réponse démographique des organes dans un modèle de fonctionnement.

La description de la plasticité en concurrence est aisée en termes de "modules". Chaque situation est à l'origine d'itinéraires différents. A partir du module initial qu'est la plantule issue de semis, une colonie va s'organiser. Deux échelles d'itinéraires de production sont à considérer : l'une concerne l'individu, l'autre la parcelle.

L'organisation d'un individu de trèfle violet est sous la dépendance du nombre d'individus de sa propre espèce qui l'entourent. A 100 plantes $/ \mathrm{m}^{2}$, l'individu élabore beaucoup de biomasse (matière sèche) constituée surtout par les limbes et les tiges. L'individu est très ramifié, ses feuilles sont nombreuses. Quoiqu'ayant une durée de vie plus longue, les feuilles naissent plus tôt que lorsque la densité est élevée.

L'augmentation de densité induit une simplification de l'organisation qui peut aller jusqu'à la disparition de l'individu; la biomasse est constituée en grande partie par des limbes et des pétioles. Il y a peu de ramifications (4 à 6 fois moins). Le processus de ramification est tardif et représente un maximum en position modulaire 5. Les limbes sont minces. Les feuilles naissent plus tard et meurent également plus tard, mais la durée de vie est, en moyenne, plus courte qu'à faible densité. La présence de la graminée en proportion décroissante (correspondant à une proportion croissante de légumineuse) agit comme la densité sur l'organisation individuelle.

En ce qui concerne le climat, il apparaît que les variables à prendre en compte sont principalement celles concernant la température. Pour le moment, on a 
étudié les paramètres d'un individu «type "; cette approche est nettement insuffisante pour rendre compte de la structure du peuplement. Cependant, il semble possible d'entamer la phase suivante de cette recherche : la modélisation de la production primaire et, a fortiori, agronomiquement utilisable. Grâce à l'acquis sur la dynamique de l'organisation d'une plante ou morphogenèse, on aboutira à des modèles non plus descriptifs mais incluant des hypothèses sur les mécanismes ce qui accroîtra considérablement leur valeur.

La production parcellaire est la résultante de la biomasse individuelle. Après l'expansion végétative de la $1^{\text {re }}$ année, le nombre d'individus baisse et tend, pour toutes les parcelles, vers une densité unique, ce qui réduit les différences de structure entre les individus moyens des densités extrêmes. Les concurrences intra et interspécifique se traduisent par des interfërences biologiques de type coopération ou compétition. Le ray-grass d'Italie est notamment avantagé lorsqu'il y a une forte densité. Ceci signifie tout simplement qu'il est d'autant plus avantagé qu'il y a plus de trèfle. Puisque la fertilisation azotée représente peu pour la graminée, l'avantage qu'elle tire laisse croire à un gain d'azote. Cette éventualité reste à démontrer.

Il semble que l'augmentation de densité soit mieux ressentie par le ray-grass d'Italie que par le trèfle violet du fait de son port dressé. Il conserve dans ces conditions une possibilité d'accès à la lumière. Il est certain que l'azote accentuerait ce résultat en permettant l'expression maximum de la morphogenèse de la graminée, compte tenu de la lumière (et donc du carbone) qu'il est en mesure de capter. Ceci souligne un aspect essentiel du phénomène de concurrence non abordé directement dans ces 2 articles. C'est que les lois de la morphogenèse établies ici sont le reflet d'une organisation potentielle (en l'absence de concurrence), organisation contrôlée génétiquement, et modulées par les disponibilités en ressources $(\mathrm{N}$ et $\mathrm{C}$ ) nécessaires à la réalisation de la morphogenèse. La prise en compte des aspects nutritifs ( $\mathrm{N}$ et photosynthèse), qui ne sont pas l'objet de la présente étude, sinon indirectement par le biais de la densité, est indispensable à une réelle intégration de la démarche morphogénétique dans une étude plus fonctionnelle des phénomènes de concurrence.

Le type de recherche présenté ici comporte quelques difficultés. Ainsi, dans l'une des expériences, 360 modules ont été suivis pour chacune des cohortes étudiées ce qui représente 13000 feuilles et nécessite l'utilisation d'un ordinateur pour l'obtention des paramètres démographiques,à la suite de l'exploitation des données. L'exploitation la plus complète possible de ces données et notamment l'approfondissement des aspects " modélisation " nécessitent de préciser encore l'organisation du trèfle violet au cours de sa croissance en association et, en particulier, la réponse de l'itinéraire morphologique dans des conditions environnementales contrôlées. Il s'agit là d'une contribution à la connaissance du « fonctionnement des agroécosystèmes prairiaux ", intégrant des concepts écologiques dans une démarche agronomique. L'originalité de l'approche démographique réside dans la possibilité de ne plus considérer la plante comme une « boîte noire ", sans structure, mais à tenir compte de sa plasticité morphogénétique. Ceci impliquant, nécessairement, et à titre complémentaire, des études de type trophique ( $\mathrm{C}$ et $\mathrm{N})$ sur les 2 partenaires de l'association. En fin de compte, on devrait arriver à coupler 2 sous-modèles : l'un morphogénétique, l'autre trophique, ce dernier prenant en compte les disponibilités en ressource.

\section{RÉFÉRENCES BIBLIOGRAPHIQUES}

\begin{abstract}
Chalamet A., Audergon J. M., Maitre J. P., 1983. Concurrence entre une graminée (Lolium multiflorum Lamk.) et une légumineuse (Trifolium pratense L.) : utilisation du ${ }^{15} \mathrm{~N}$ pour l'étude de la nutrition azotée. Acta Oecol. Oecol. Plant., 4 (18), 2, 12-33.

Guy P., 1964. Compte rendu d'une expérience préliminaire sur la croissance et le développement des luzernes. Ann. Amélior. Plantes, 14 (4), 383-417.

Jacquard P., 1975. Concurrence intraspécifique et potentialité de rendement. Ann. Amélior. Plantes, 25 (1), 3-24.

Jacquard P., 1977. Relations entre espèces dans les associations graminée-légumineuse. Le Sél. français, 24, 3-28.
\end{abstract}

Reç le 20 avril 1984. Accepté le 12 novembre 1984.
Maitre J. P., Assemat L., Jacquard P., 1985. Croissance en association du trèfle violet (Trifolium pratense L.) avec du ray-grass d'Italie (Lolium multiflorum Lam. ssp. ilalicum) : I. Description de l'organisation morphologique du trèfle. Agronomie, 5 (3).

Wacquant J. P., El-Chahata H., Jacquard P., 1981. Effect of mineral stress on competition and associated growth of a grass (Lolium italicum L.) and a legume (Trifolium pratense L.). In C.E. Wright : "Plant physiology and herbage production ». Occasional Symp. N $\mathrm{N}^{\circ}$ 13, Br. Grassi. Soc., 231-234. 